\title{
Discovery of Oxazolidinone-Based Heterocycles as Subtype Selective Sigma-2 Ligands
}

\section{Benjamin E Blass ( $\nabla$ tud12939@temple.edu )}

Temple University School of Pharmacy https://orcid.org/0000-0003-2449-4503

\section{Richie Rashmin Bhandare}

Ajman University of Science and Technology College of Pharmacy and Health Science

\section{Daniel J. Canney}

Temple University School of Pharmacy

\section{Research Article}

Keywords: Alzheimer's disease, sigma-2, sigma-1, sigma receptor, oxazolidin-2-one

Posted Date: October 29th, 2021

DOl: https://doi.org/10.21203/rs.3.rs-977859/v1

License: (1) This work is licensed under a Creative Commons Attribution 4.0 International License.

Read Full License

Version of Record: A version of this preprint was published at Medicinal Chemistry Research on January 24th, 2022. See the published version at https://doi.org/10.1007/s00044-022-02848-4. 
Discovery of oxazolidinone-based heterocycles as subtype selective sigma-2 ligands

Benjamin E. Blass ${ }^{\mathrm{a} .}$ Richie Rashmin Bhandare, ${ }^{\mathrm{b}}$ and Daniel J. Canney ${ }^{\mathrm{a}}$

$\varangle$ Benjamin E. Blass

Benjamin.Blass@Temple.edu

Richie Rashmin Bhandare

r.bhandareh@ajman.ac.ae

Daniel J. Canney

canney@temple.edu

${ }^{a}$ Temple University School of Pharmacy, 3307 North Broad Street, Philadelphia, PA 194140

USA

${ }^{\mathrm{b}}$ Ajman University, College of Pharmacy, Department of Pharmaceutical Sciences, P.O. Box 346, Ajman UAE 
Abstract: Alzheimer's disease is a major, unmet medical need that impacts 6 million people in the US alone. Therapeutic options are limited, and the root cause of this condition remains unclear. The Amyloid Hypothesis has been proposed as a means of explaining the formation of amyloid plaques in the brain of patient. The sigma-2 receptor was recently identified as a potential therapeutic target capable of arresting the formation of amyloid plaques. Herein, we report the identification of a series of novel, functionalized oxazolidin-2-ones sigma-2 ligands.

\section{Graphical Abstract:}

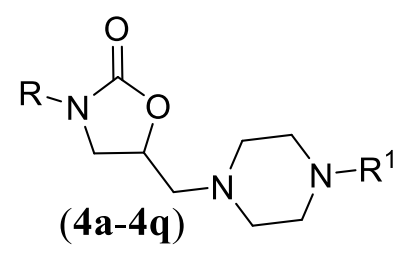

Keywords: Alzheimer's disease, sigma-2, sigma-1, sigma receptor, oxazolidin-2-one

Introduction: Alzheimer's disease remains a major, unmet medical need despite decades of intensive research. Originally described in 1907 by the German psychiatrist Alois Alzheimer, this neurodegenerative disease leads inevitably to substantial, debilitating cognitive decline.[1] According to the Alzheimer's Association, there are over 6 million people in the US alone suffering from this condition, and it is anticipated that the patients count will increase to over 13 million by 2050. In addition, the cost of care for Alzheimer's disease patients and related dementias in the US exceeds $\$ 355$ billion and is expected to rise to $\sim \$ 1.1$ trillion by 2050.[2] To date, there are no approved therapeutic capable of slowing disease progression, and despite decades of research, the root cause of Alzheimer's disease remains a mystery.

Extensive research efforts have, however, linked Alzheimer's disease progression to the formation of $\beta$-amyloid plaques and neurofibrillary tangles in the cortical and subcortical regions 
of the brain, a theory known as the Amyloid Hypothesis. The formation of these plaques begins with sequential cleavage of the amyloid precursor protein (APP) by $\beta$-secretase 1 (BACE1) and $\gamma$-secretase. APP is initially cleaved by BACE1 to produce the soluble peptide $\beta$-APP and a membrane bound peptide designated C-99. Further processing of C-99 by $\gamma$-secretase produces $A \beta 42$, which initially oligomerizes to form $A \beta$ oligomers ( $A \beta O)$, Aggregation of $A \beta O$ s produces $\beta$-amyloid plaques, a hallmark feature of Alzheimer's disease.[3] However, clinical trials targeting this pathway via small molecule such as verubecestat (1)[4] and semagacestat (2)[5], as well as monoclonal antibodies such as Bapineuzumab and Solanezuma, have ended in failure.[6] The lone exception to this series of failed Alzheimer's disease drug discovery programs is the antibody, Aducanumab, whose FDA approval remains controversial.[7]

A recently proposed modification of the Amyloid Hypothesis proposes that the A $\beta O s$ formed as precursors to amyloid plaques are a key player in disease progression. This new aspect of the Amyloid Hypothesis is based in part on the observation that $\mathrm{A} \beta \mathrm{O}$ s are the most potent neurotoxic structural form of the $A \beta$ protein.[8] In addition, the A673T mutation (the Icelandic mutation), which is the only known protective mutation lowers the incidence of Alzheimer's disease, has been demonstrated to produce structural changes in $A \beta$ proteins [9] that significantly lower the binding affinity of $A \beta O$ s at synaptic receptors.[10] Several proteins have been proposed as $\mathrm{A} \beta \mathrm{O}$ receptors including the $\alpha 7$ nicotinic

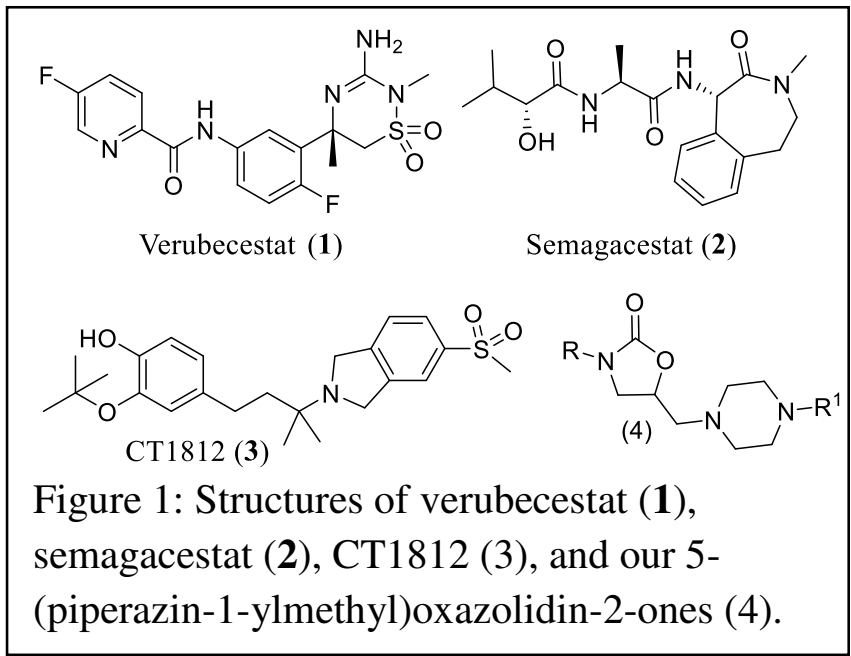


acetylcholine receptor, the P75 neurotrophin receptor, $\beta$-adrenergic receptors, (PrPC), PirB/LilrB2, , PrPC, mGluR5, Fc $\gamma$ RIIb, EphB2,[11], and the Sigma-2 receptor (б2).

The nature and function of $\sigma 2$, has been the subject of intense research since it was originally characterized by W. D. Brown et.al. in 1993.[12] Several lines of evidence suggest that $\sigma 2$ is present in lysosomes and the endoplasmic reticulum (ER), that cholesterol binds to this receptor,[13] and that $\sigma 2$ plays a role in the regulation of the Niemann-Pick protein NPC1.[14] In 2017, A.C. Krusea et. al. conclusively demonstrated that the protein originally described as the $\sigma_{2}$ receptor is identical to Transmembrane Protein 97 (TMEM97, also known as MAC30 (Meningioma-associated protein).[15] Separately, scientist at Cognition Therapeutics (Pittsburgh, $\mathrm{PA}$ ) recently demonstrated that $\mathrm{A} \beta \mathrm{O}$ binding to neuronal receptors is modulated by $\sigma 2$ and that small molecules targeting this receptor protect neurons from the synaptotoxc effects of AßOs.[16] Their efforts lead to the identification of CT1812, a potent $\sigma 2$ ligand $(\mathrm{Ki}=8.5 \mathrm{nM})$, that is efficacious in the hAPP Swe/Ldn mouse model and is currently undergoing clinical trials as a potential Alzheimer's disease therapy.[17]

We have recently become interested in the identification of novel $\sigma 2$ ligand based on their potential utility as Alzheimer's disease therapy, as well as opportunities in traumatic brain injury,[18] neuropathic pain,[19] schizophrenia,[20] and cancer.[21] As part of this effort, we

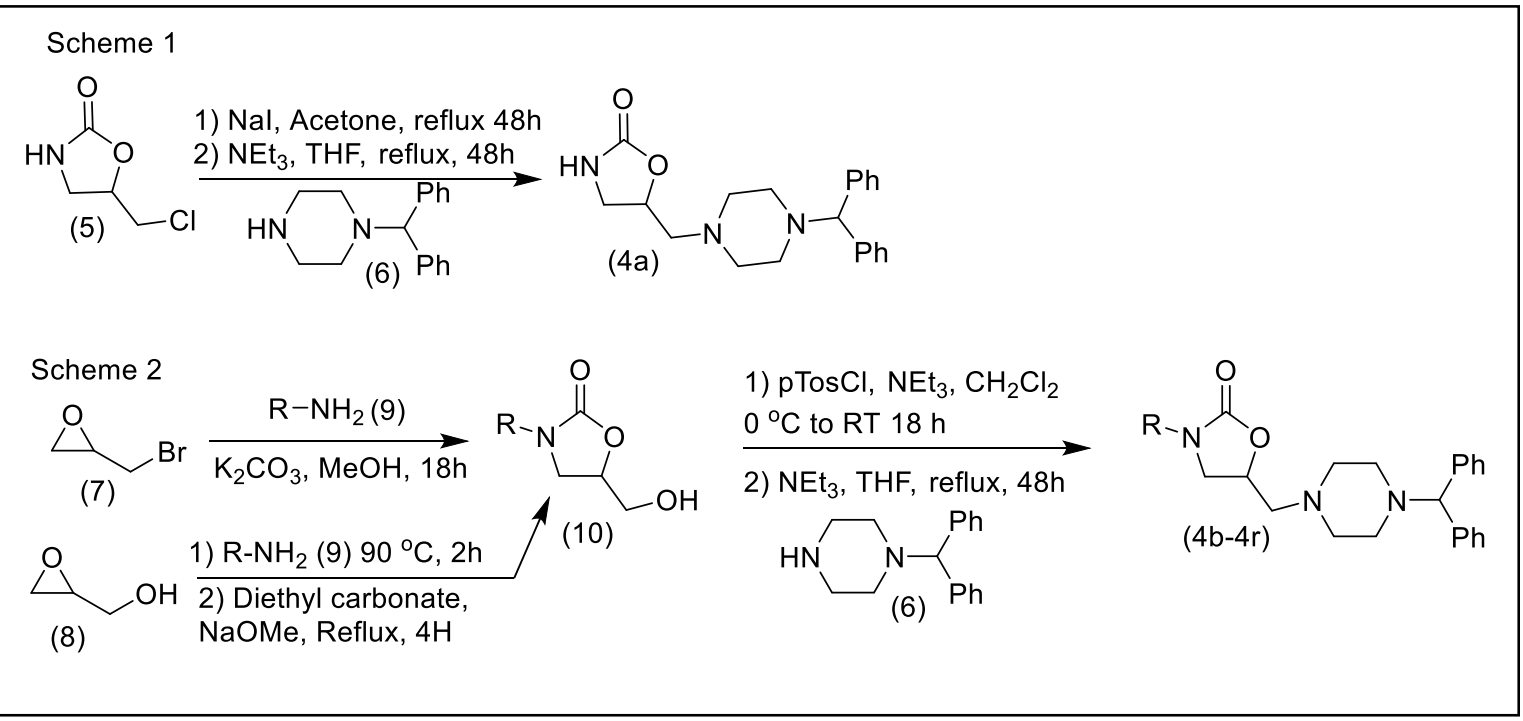


have been exploring the chemical space associated with a series of 5-(piperazin-1ylmethyl)oxazolidin-2-one (4). Preliminary assessment of initial compounds prepared in this series by the Psychoactive Drug Screening Program (PDSP) demonstrated that members of this family that are potent $\sigma_{2}$ binders with varying levels of $\sigma_{1} / \sigma_{2}$ selectivity. Given the therapeutic potential of this receptor, a drug discovery program focused on the identification of novel, druglike functionalized 5-(piperazin-1-ylmethyl)oxazolidin-2-ones with potent $\sigma_{2}$ binding was established. The synthesis, characterization and preliminary evaluation of these compounds as potential selective $\sigma_{2}$ ligands will be presented.

Results and discussion: Synthesis of the 5-(piperazin-1-ylmethyl)oxazolidin-2-ones was conducted using the methods described in schemes 1 and 2. The unsubstituted oxazolidin-2-ones (4a) was prepared from the known primary chloride (5). Conversion of this starting material to the corresponding iodide using $\mathrm{NaI}$ in acetone was followed by displacement of the iodide with (6) to provide (4a). Synthesis of the remaining compounds (4b-4r) began with either epibromohydrin (7) or Glycidol (8). Reaction of (7) with an amine (9) in methanolic K2CO3 provided oxazolidin-2-one (10). Alternatively, reaction of $(\mathbf{8})$ with an amine (9), followed by ring closure with diethyl carbonate in the presence of sodium methoxide provided (10). Conversion of (10) to the corresponding tosylate ( $\mathrm{pTosCl}, \mathrm{NEt}_{3}, \mathrm{THF}$ ), followed by displacement of the leaving group with an amine (9) provided the final target compounds (4b-4r).

Table 1 includes the in vitro binding $\left(\mathrm{K}_{\mathrm{i}}\right.$ at $\sigma 1$ and $\left.\sigma 2\right)$ as well as the physicochemical properties (MW, TPSA, LogP) of target compounds. The compounds prepared and tested have TPSA and MW values that suggest they will cross the BBB, and all but cLogP values are higher than the desired range of 2-4 for BBB penetration. The structure-activity relationship began with the unsubstituted oxazolidin-2-one (4a), which demonstrated minimal affinity for $\sigma 1$ and $\sigma 2\left(\mathrm{~K}_{\mathrm{i}}\right.$ 
$=10,000 \mathrm{nM}$ at both $\sigma 1$ and $\sigma 2)$. Capping of the amide with a 3 to 6 membered cycloalkane (4b4e) or a benzene ring (4f) produced compounds with moderate $\sigma 2$ binding affinity $\left(\mathrm{K}_{\mathrm{i}}=116\right.$ to $530 \mathrm{nM})$ that had limited capacity to bind to $\sigma 1\left(\mathrm{~K}_{\mathrm{i}}=10,000 \mathrm{nM}\right)$. Interestingly, the impact of a benzyl substituent on $\sigma 2$ affinity was highly dependent on the electronic character of the benzene ring. In the absence of a substituent, the benzyl derivative $(\mathbf{4 g})$ is approximately twice as potent at $\sigma 2\left(\mathrm{~K}_{\mathrm{i}}=91 \mathrm{nM}\right)$ as the corresponding phenyl derivative (4f), and the high degree of selectivity over $\sigma 1$ is maintained $\left(\mathrm{K}_{\mathrm{i}}=10,000 \mathrm{nM}\right) . \sigma 2$ affinity increased three-fold when a 4-F substituent was appended to the benzene ring $\left(\mathbf{4 h}, \sigma 2 \mathrm{~K}_{\mathrm{i}}=36 \mathrm{nM}\right)$, and high degree of selectivity over $\sigma 1$ $\left(\mathrm{K}_{\mathrm{i}}=2,847 \mathrm{nM}\right)$ was maintained. In contrast, incorporation of the electron donating 4-OMe (4i) or 4-Mr (4j) produced compounds that had limited capacity to bind both sigma receptors ( $\sigma 2$ and $\left.\sigma 1 \mathrm{~K}_{\mathrm{i}}=10,000 \mathrm{nM}\right)$. Replacing the benzene ring of $(\mathbf{4 g})$ with either a cyclohexane ring $(4 \mathrm{k})$ or tetrahydropyran ring (4I) also lead to a substantial drop in $\sigma 2$ affinity $(\mathrm{Ki}=1379$ and $2428 \mathrm{nM}$ respectively). $\sigma 2$ binding affinity and selectivity over $\sigma 1$ were maintained when the benzyl group (4g) was extended by an additional methylene $(\mathbf{4 m}, \sigma 2 \mathrm{Ki}=49 \mathrm{nM}, \sigma 1 \mathrm{Ki}=10,000 \mathrm{nM})$, but incorporation of both electron withdrawing (4n) and electron donating substituents $(\mathbf{4 o}, \mathbf{4 p})$ led to a significant loss in $\sigma 2$ binding affinity, as did the addition of a third methylene unit (4q).

Follow-up assessment of our most potent $\sigma 2$ ligand (4h) at a series of key CNS targets available through the PDSP demonstrated that this compound is selective for $\sigma 2$ over a variety of important CNS targets. Specifically, (4h) is $\geq 100$-fold selective for $\sigma 2$ over, $\alpha_{1 \mathrm{~B}}, \alpha_{1 \mathrm{D}}, \alpha_{2 \mathrm{~A}}$, the benzodiazepine receptor (brain and peripheral), $\beta_{1}, \beta_{2}, \beta_{3} \mathrm{D}_{1}, \mathrm{D}_{5}, \mathrm{GABA}-\mathrm{A}, \mathrm{H}_{2}, \mathrm{H}_{3}, 5-\mathrm{HT}_{1 \mathrm{~A}}, 5-$ $\mathrm{HT}_{1 \mathrm{~B}}, 5-\mathrm{HT}_{1 \mathrm{D}}, 5-\mathrm{HT}_{1 \mathrm{E}},, 5-\mathrm{HT}_{2 \mathrm{C}}, \mathrm{M}_{1}, \mathrm{M}_{2}, \mathrm{M}_{3}, \mathrm{M}_{4}, \mathrm{M}_{5}$, the $\delta$-opioid receptor, the $\mu$-Opioid receptor, and the serotonin transporter. In addition, (4h) was $\geq 25$ fold $\alpha_{1 \mathrm{~A}}, \alpha_{2 \mathrm{~B}}, \alpha_{2 \mathrm{C}}, \mathrm{D}_{2}, \mathrm{D}_{3}, 5$ - 
$\mathrm{HT}_{2 \mathrm{~A}}$, the $\kappa$-opioid receptor, and the norepinephrine transporter. Less selectivity was observed over $\mathrm{D}_{4}(521 \mathrm{nM}), 5 \mathrm{HT}_{2 \mathrm{~b}}(201 \mathrm{nM})$, DAT $(186 \mathrm{nM})$, and $\mathrm{H}_{1}(99 \mathrm{nM})$,

Conclusion: In summary, we have identified a series of 5-(piperazin-1ylmethyl)oxazolidin-2-ones (4) $\sigma 2$ ligand that are highly selective for this receptor over $\sigma 1$. In addition, we have established preliminary SAR that could be used to extend our exploration of this series and identified a novel compound (4h) that is selective for $\sigma 2$ over a range of key CNS targets. Future efforts on determining in vitro ADME properties (e.g. solubility, CYP450 inhibition, microsomal stability) of our compounds and exploring the impact of additional substituents on the embedded benzene rings.

Experimental methods and materials: Reagents were purchased from Fisher Scientific, VWR International, Sigma Aldrich, and Combi-Blocks, Inc. Chromatographic purification of compounds (normal phase and reverse phase) was carried out on a Teledyne Isco Combiflash RF system. H-NMR spectra were obtained on a Bruker 400-MHz NMR. Chemical shift values $(\delta$ values) were reported in ppm relative to TMS. For multiplicity, $\mathrm{s}=$ singlet, $\mathrm{d}=$ doublet, $\mathrm{t}=$ triplet, $\mathrm{m}=$ multiplet. Purity $(\%)$ and mass spectral data were determined with a Waters Agilent $1200 \mathrm{HPLC}$ MS (Zorbax SB-C18, $2.1 \times 30 \mathrm{~mm}, 3.5 \mu \mathrm{m}, 100 \%$ water/0.1\% formic acid to $100 \%$ acetonitrile $/ 0.1 \%$ formic acid over 4.0 minutes, $1.0 \mathrm{~mL} / \mathrm{min}$.) with a diode array detector from 210-400 nm and Agilent 6130 quadrupole MS. All compounds were purified to 95\% purity or greater as determined by HPLC/MS and 1H-NMR. Melting points were recorded on a capillary melting point apparatus.

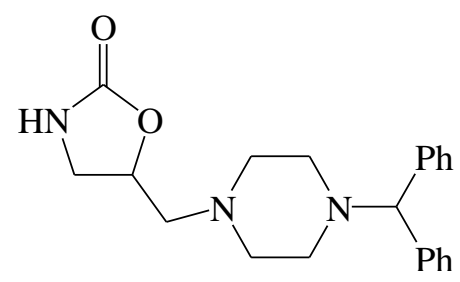


5-(4-Benzhydryl-piperazin-1-ylmethyl)-oxazolidin-2-one (4a): Sodium iodide (13.19 g, 88.52 $\mathrm{mmol})$ in acetone $(35 \mathrm{~mL})$ was added to a round bottom flasks containing 5-Chloromethyloxazolidin-2-one $(0.8 \mathrm{~g}, 5.90 \mathrm{mmol})$ and the mixture was refluxed for 48 hours. The reaction was later quenched with water and extracted with ether. The organic layer was washed with water, brine and dried over $\mathrm{MgSO}_{4}$ to obtain the product as a light-yellow solid that was dissolved in THF $\mathrm{mL}$ ) that contained diphenylmethylpiperazine $(2.43 \mathrm{gm}, 9.629 \mathrm{mmol})$ and triethyl amine (1.299 gm, $12.83 \mathrm{mmol})$. The reaction was stirred at reflux under a nitrogen atmosphere for 48 hours. The mixture was filtered (when appropriate), concentrated under reduced pressure and the residue was dissolved in dichloromethane and purified by flash silica gel chromatography using methanol (0-10\%) in dichloromethane and reverse phase chromatography using acetonitrile: water $(0-100 \%)$ to obtain the desired product (0.203 gm, $17.99 \%, 2$ steps). 1H-NMR \{CDCl3, $400 \mathrm{MHz}, \delta(\mathrm{ppm})\} 2.32-2.52(\mathrm{~m}, 8 \mathrm{H}), 2.59-2.64(\mathrm{~m}, 1 \mathrm{H}), 3.21(\mathrm{t}, 1 \mathrm{H}), 3.50(\mathrm{t}, 1 \mathrm{H}), 4.13(\mathrm{~s}$, 1H), $4.61-4.68(\mathrm{~m}, 1 \mathrm{H}), 6.08(\mathrm{~s}, 1 \mathrm{H}), 7.06-7.10(\mathrm{~m}, 2 \mathrm{H}), 7.16-7.19(\mathrm{~m}, 4 \mathrm{H}), 7.31-7.33(\mathrm{~m}$, 4H). LC - MS (ESI) (m/z) $352.2(\mathrm{M}+1)+$.

Examples (4b), (4c), (4d), and (4e) were prepared according to previously published methods.[22] (2-oxo-3-phenyloxazolidin-5-yl)methyl 4-methylbenzenesulfonate was prepared according to previously published methods [23]

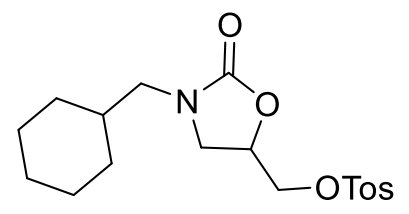

(3-(cyclohexylmethyl)-2-oxooxazolidin-5-yl)methyl 4-methylbenzenesulfonate: To a suspension of $\mathrm{K}_{2} \mathrm{CO}_{3}(2.5 \mathrm{gm}, 18.08 \mathrm{mmol})$ in anhydrous methanol $(30 \mathrm{ml})$ containing epibromohydrin (4.965 gm, $36.24 \mathrm{mmol}$ ) was added the cyclohexyl methylamine (4.089 gm, $36.12 \mathrm{mmol}$ ) and the reaction was stirred overnight. The reaction mixture was later filtered and the organic solvent 
was stripped off under reduced pressure to obtain a liquid residue. It was utilized for the tosylation step without purification.

p-Toluene sulfonyl chloride $(13.5 \mathrm{gm}, 7.096 \mathrm{mmol})$ in methylene chloride $(50 \mathrm{ml})$ was added dropwise to a chilled solution of crude alcohol (7.5756 gm, $35.52 \mathrm{mmol})$ and triethylamine (7.1874 gm, $71.028 \mathrm{mmol})$ in methylene chloride $(60 \mathrm{ml})$. The reaction was stirred in ice at $0{ }^{\circ} \mathrm{C}$ for $1 \mathrm{hr}$ followed by overnight stirring at room temperature. It was later quenched with ice water and the organic layer was washed successfully with $10 \% \mathrm{HCl}$, saturated aqueous sodium bicarbonate and brine. The organic layer was dried over $\mathrm{MgSO}_{4}$ and concentrated under reduced pressure to obtain a solid/oil which was purified on silica gel redisep column using hexane: ethyl acetate $(0-100 \%)$ to obtain the desired product (2.9782 gm, $22.81 \%$ yield, 2 steps). ${ }^{1} \mathrm{H}-\mathrm{NMR}$ $\left\{\mathrm{CDCl}_{3}, 400 \mathrm{MHz}, \delta(\mathrm{ppm})\right\} 0.89-0.99(\mathrm{~m}, 2 \mathrm{H}), 1.11-1.28(\mathrm{~m}, 3 \mathrm{H}), 1.47-1.61(\mathrm{~m}, 1 \mathrm{H}), 1.63$ $-1.74(\mathrm{~m}, 6 \mathrm{H}), 2.47(\mathrm{~s}, 3 \mathrm{H}), 3.00-3.11(\mathrm{~m}, 2 \mathrm{H}), 3.42-3.46(\mathrm{~m}, 1 \mathrm{H}), 3.63(\mathrm{t}, 1 \mathrm{H}), 4.11-4.18$ $(\mathrm{m}, 2 \mathrm{H}), 4.66-4.71(\mathrm{~m}, 1 \mathrm{H}), 7.35-7.39(\mathrm{~m}, 2 \mathrm{H}), 7.79-7.81(\mathrm{~m}, 2 \mathrm{H}) . \mathrm{LC}-\mathrm{MS}(\mathrm{ESI})(\mathrm{m} / \mathrm{z})$ $368.1(\mathrm{M}+1)^{+}$.

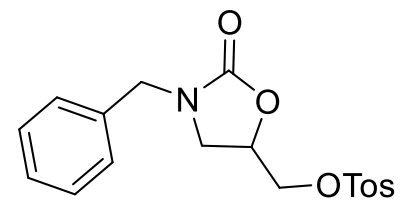

(3-benzyl-2-oxooxazolidin-5-yl)methyl 4-methylbenzenesulfonate was prepared according to the procedure of (3-(cyclohexylmethyl)-2-oxooxazolidin-5-yl)methyl 4-methylbenzenesulfonate except cyclohexyl methylamine was replaced with benzyl amine. Yield: $68.68 \%$. ${ }^{1} \mathrm{H}-\mathrm{NMR}$ $\left\{\mathrm{CDCl}_{3}, 400 \mathrm{MHz}, \delta(\mathrm{ppm})\right\} 2.45(\mathrm{~s}, 3 \mathrm{H}), 3.23-3.27(\mathrm{~m}, 1 \mathrm{H}), 3.48(\mathrm{t}, 1 \mathrm{H}), 4.07-4.14(\mathrm{~m}, 1 \mathrm{H})$, $4.39(\mathrm{~s}, 2 \mathrm{H}), 4.62-4.68(\mathrm{~m}, 1 \mathrm{H}), 7.24-7.26(\mathrm{~m}, 2 \mathrm{H}), 7.30-7.38(\mathrm{~m}, 5 \mathrm{H}), 7.73-7.76(\mathrm{~m}, 2 \mathrm{H})$. LC - MS (ESI) (m/z) $363.1(\mathrm{M}+1)^{+}$. 
<smiles>O=C1OC(CO[Se+])CN1Cc1ccc(F)cc1</smiles>

(3-(4-fluorobenzyl)-2-oxooxazolidin-5-yl)methyl 4-methylbenzenesulfonate was prepared according to the procedure of (3-(cyclohexylmethyl)-2-oxooxazolidin-5-yl)methyl 4methylbenzenesulfonate except cyclohexyl methylamine was replaced with 4-fluorobenzyl amine. Yield: $48.95 \% .{ }^{1} \mathrm{H}-\mathrm{NMR}\left\{\mathrm{CDCl}_{3}, 400 \mathrm{MHz}, \delta(\mathrm{ppm})\right\} 2.48(\mathrm{~s}, 3 \mathrm{H}), 3.28-3.30(\mathrm{~m}, 1 \mathrm{H})$, $3.50(\mathrm{t}, 1 \mathrm{H}), 4.11-4.12(\mathrm{~m}, 2 \mathrm{H}), 4.34-4.43(\mathrm{~m}, 2 \mathrm{H}), 4.64-4.70(\mathrm{~m}, 1 \mathrm{H}), 7.03-7.07(\mathrm{~m}, 2 \mathrm{H})$, $7.22-7.27(\mathrm{~m}, 2 \mathrm{H}), 7.38-7.39(\mathrm{~m}, 2 \mathrm{H}), 7.76-7.78(\mathrm{~m}, 2 \mathrm{H})$. LC - MS (ESI) (m/z) 380.1 $(\mathrm{M}+1)^{+}$<smiles>COc1ccc(CN2CC(C[OH2+])OC2=O)cc1</smiles>

(3-(4-methoxybenzyl)-2-oxooxazolidin-5-yl)methyl 4-methylbenzenesulfonate was prepared according to the procedure of (3-(cyclohexylmethyl)-2-oxooxazolidin-5-yl)methyl 4methylbenzenesulfonate except cyclohexyl methylamine was replaced with 4-methoxybenzyl amine. Yield: $79.72 \% .{ }^{1} \mathrm{H}-\mathrm{NMR}\left\{\mathrm{CDCl}_{3}, 400 \mathrm{MHz}, \delta(\mathrm{ppm})\right\} 2.46(\mathrm{~s}, 3 \mathrm{H}), 3.44(\mathrm{t}, 1 \mathrm{H}), 3.81(\mathrm{~s}$, $3 \mathrm{H}), 4.04-4.13(\mathrm{~m}, 2 \mathrm{H}), 4.33(\mathrm{~s}, 1 \mathrm{H}), 4.60-4.66(\mathrm{~m}, 1 \mathrm{H}), 6.86-6.90(\mathrm{~m}, 2 \mathrm{H}), 7.15-7.19(\mathrm{~m}$, 2H), $7.35-7.37(\mathrm{~m}, 2 \mathrm{H}), 7.74-7.76(\mathrm{~m}, 2 \mathrm{H}) . \mathrm{LC}-\mathrm{MS}(\mathrm{ESI})(\mathrm{m} / \mathrm{z}) 392.1(\mathrm{M}+1)^{+}$.<smiles>Cc1ccc(CN2CC(C[OH2+])OC2=O)cc1</smiles> 
(3-(4-methylbenzyl)-2-oxooxazolidin-5-yl)methyl 4-methylbenzenesulfonate was prepared according to the procedure of (3-(cyclohexylmethyl)-2-oxooxazolidin-5-yl)methyl 4methylbenzenesulfonate except cyclohexyl methylamine was replaced with 4-methylbenzyl amine. Yield: $64.76 \% .{ }^{1} \mathrm{H}-\mathrm{NMR}\left\{\mathrm{CDCl}_{3}, 400 \mathrm{MHz}, \delta(\mathrm{ppm})\right\} 2.27$ (s, 3H), $2.38(\mathrm{~s}, 3 \mathrm{H}), 3.15-$ $3.19(\mathrm{~m}, 1 \mathrm{H}), 3.38(\mathrm{t}, 1 \mathrm{H}), 3.96-4.04(\mathrm{~m}, 2 \mathrm{H}), 4.23-4.32(\mathrm{~m}, 2 \mathrm{H}), 4.52-4.58(\mathrm{~m}, 1 \mathrm{H}), 7.02-$ $7.09(\mathrm{~m}, 4 \mathrm{H}), 7.28(\mathrm{~d}, 2 \mathrm{H}), 7.67-7.69(\mathrm{~m}, 2 \mathrm{H})$. LC - MS (ESI) (m/z) $376.1(\mathrm{M}+1)^{+}$.

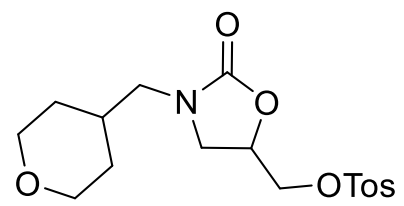

Toluene-4-sulfonic acid 2-oxo-3-(tetrahydro-pyran-4-ylmethyl)-oxazolidin-5-ylmethyl ester was prepared according to the procedure of (3-(cyclohexylmethyl)-2-oxooxazolidin-5-yl)methyl 4methylbenzenesulfonate except cyclohexyl methylamine was replaced with (tetrahydro-2Hpyran-4-yl)methanamine. Yield: $63.63 \% .{ }^{1} \mathrm{H}-\mathrm{NMR}\left\{\mathrm{CDCl}_{3}, 400 \mathrm{MHz}, \delta(\mathrm{ppm})\right\} 1.23-1.36$ (m, 2H), $1.54-1.58(\mathrm{~m}, 2 \mathrm{H}), 1.78-1.89(\mathrm{~m}, 1 \mathrm{H}), 2.45(\mathrm{~s}, 3 \mathrm{H}), 3.01-3.06(\mathrm{~m}, 1 \mathrm{H}), 3.14-3.19$ (m, 1H), $3.32-3.38(\mathrm{~m}, 2 \mathrm{H}), 3.46-3.50(\mathrm{~m}, 1 \mathrm{H}), 3.66(\mathrm{t}, 1 \mathrm{H}), 3.93-3.97(\mathrm{~m}, 2 \mathrm{H}), 4.15(\mathrm{~d}$, 2H), $4.66-4.74(\mathrm{~m}, 1 \mathrm{H}), 7.37(\mathrm{~d}, 2 \mathrm{H}), 7.77(\mathrm{~d}, 2 \mathrm{H})$. LC - MS (ESI) (m/z) $370.1(\mathrm{M}+1)^{+}$.

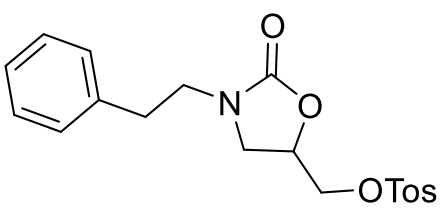

(2-oxo-3-phenethyloxazolidin-5-yl)methyl 4-methylbenzenesulfonate was prepared according to the procedure of (3-(cyclohexylmethyl)-2-oxooxazolidin-5-yl)methyl 4-methylbenzenesulfonate except cyclohexyl methylamine was replaced with phenylethylamine. Yield: $81.75 \%$. ${ }^{1} \mathrm{H}$ NMR $\left\{\mathrm{CDCl}_{3}, 400 \mathrm{MHz}, \delta(\mathrm{ppm})\right\} 2.46(\mathrm{~s}, 3 \mathrm{H}), 2.83(\mathrm{t}, 1 \mathrm{H}), 3.25-3.29(\mathrm{~m}, 1 \mathrm{H}), 3.45-3.52$ 
(m, 3H), $4.01-4.09(\mathrm{~m}, 2 \mathrm{H}), 4.56-4.61(\mathrm{~m}, 1 \mathrm{H}), 7.20-7.26(\mathrm{~m}, 3 \mathrm{H}), 7.30-7.33(\mathrm{~m}, 2 \mathrm{H})$, $7.38(\mathrm{~d}, 2 \mathrm{H}), 7.77-7.80(\mathrm{~m}, 2 \mathrm{H}) . \mathrm{LC}-\mathrm{MS}(\mathrm{ESI})(\mathrm{m} / \mathrm{z}) 376.1(\mathrm{M}+1)^{+}$.

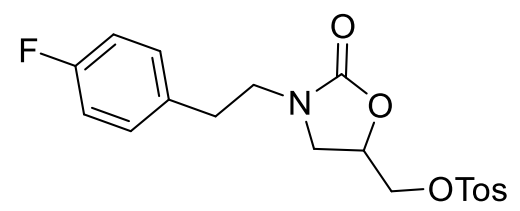

(3-(4-fluorophenethyl)-2-oxooxazolidin-5-yl)methyl 4-methylbenzenesulfonate was prepared according to the procedure of (3-(cyclohexylmethyl)-2-oxooxazolidin-5-yl)methyl 4methylbenzenesulfonate except cyclohexyl methylamine was replaced with 4fuorophenylethylamine. Yield: $61.11 \% .{ }^{1} \mathrm{H}-\mathrm{NMR}\left\{\mathrm{CDCl}_{3}, 400 \mathrm{MHz}, \delta(\mathrm{ppm})\right\} 2.47$ (s, 3H), $2.84(\mathrm{t}, 2 \mathrm{H}), 3.32-3.36(\mathrm{~m}, 1 \mathrm{H}), 3.39-3.54(\mathrm{~m}, 3 \mathrm{H}), 4.02-4.16(\mathrm{~m}, 3 \mathrm{H}), 6.98-7.03(\mathrm{~m}, 2 \mathrm{H})$, $7.15-7.20(\mathrm{~m}, 2 \mathrm{H}) 7.39(\mathrm{~d}, 2 \mathrm{H}), 7.78-7.80(\mathrm{~m}, 2 \mathrm{H}) . \mathrm{LC}-\mathrm{MS}(\mathrm{ESI})(\mathrm{m} / \mathrm{z}) 394.1(\mathrm{M}+1)^{+}$.

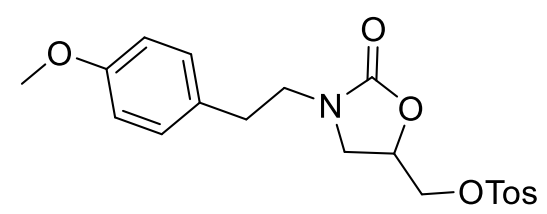

Toluene-4-sulfonic acid 3-[2-(4-methoxy-phenyl)-ethyl]-2-oxo-oxazolidin-5-ylmethyl ester was prepared according to the procedure of (3-(cyclohexylmethyl)-2-oxooxazolidin-5-yl)methyl 4methylbenzenesulfonate except cyclohexyl methylamine was replaced with 4methoxyphenylethylamine. Yield: 84.55\%. ${ }^{1} \mathrm{H}-\mathrm{NMR}\left\{\mathrm{CDCl}_{3}, 400 \mathrm{MHz}, \delta(\mathrm{ppm})\right\} 2.47(\mathrm{~s}, 3 \mathrm{H})$, $2.78(\mathrm{t}, 2 \mathrm{H}), 3.26-3.30(\mathrm{~m}, 1 \mathrm{H}), 3.42-3.52(\mathrm{~m}, 3 \mathrm{H}), 3.81(\mathrm{~s}, 3 \mathrm{H}), 4.01-4.16(\mathrm{~m}, 3 \mathrm{H}), 4.56-$ $4.64(\mathrm{~m}, 1 \mathrm{H}), 6.85(\mathrm{~d}, 2 \mathrm{H}), 7.11(\mathrm{~d}, 2 \mathrm{H}), 7.38(\mathrm{~d}, 2 \mathrm{H}), 7.78(\mathrm{~d}, 2 \mathrm{H})$. LC - MS (ESI) (m/z) 406.1 $(\mathrm{M}+1)^{+}$<smiles>Cc1ccc(CCN2CC(C[O+3])OC2=O)cc1</smiles> 
Toluene-4-sulfonic acid 2-oxo-3-(2-p-tolyl-ethyl)-oxazolidin-5-ylmethyl ester was prepared according to the procedure of (3-(cyclohexylmethyl)-2-oxooxazolidin-5-yl)methyl 4methylbenzenesulfonate except cyclohexyl methylamine was replaced with 4methylphenylethylamine. Yield: $75.91 \% .{ }^{1} \mathrm{H}-\mathrm{NMR}\left\{\mathrm{CDCl}_{3}, 400 \mathrm{MHz}, \delta(\mathrm{ppm})\right\} 2.34$ (s, 3H), $2.47(\mathrm{~s}, 3 \mathrm{H}), 2.81(\mathrm{t}, 2 \mathrm{H}), 3.26-3.30(\mathrm{~m}, 1 \mathrm{H}), 3.44-3.53(\mathrm{~m}, 3 \mathrm{H}), 4.01-4.16(\mathrm{~m}, 2 \mathrm{H}), 4.56-$ $4.62(\mathrm{~m}, 1 \mathrm{H}), 7.08-7.13(\mathrm{~m}, 4 \mathrm{H}), 7.37-7.39(\mathrm{~d}, 2 \mathrm{H}), 7.78-7.80(\mathrm{~d}, \mathrm{~d}, 2 \mathrm{H})$. LC - MS (ESI) $(\mathrm{m} / \mathrm{z}) 390.1(\mathrm{M}+1)^{+}$.

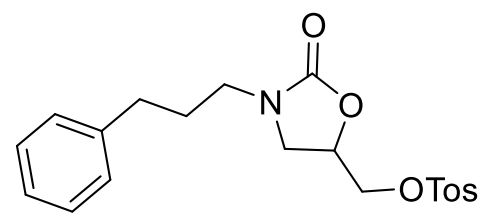

(2-oxo-3-(3-phenylpropyl)oxazolidin-5-yl)methyl 4-methylbenzenesulfonate was prepared according to the procedure of (3-(cyclohexylmethyl)-2-oxooxazolidin-5-yl)methyl 4methylbenzenesulfonate except cyclohexyl methylamine was replaced with phenylpropylamine. Yield: $76.86 \%{ }^{1} \mathrm{H}-\mathrm{NMR}\left\{\mathrm{CDCl}_{3}, 400 \mathrm{MHz}, \delta(\mathrm{ppm})\right\} 1.83-1.90(\mathrm{~m}, 2 \mathrm{H}), 2.45$ (s, 3H), $2.61-$ $2.66(\mathrm{~m}, 2 \mathrm{H}), 3.25-3.32(\mathrm{~m}, 2 \mathrm{H}), 3.38-3.42(\mathrm{~m}, 1 \mathrm{H}), 3.58(\mathrm{t}, 1 \mathrm{H}), 4.11-4.13(\mathrm{~m}, 2 \mathrm{H}), 4.59-$ $4.65(\mathrm{~m}, 1 \mathrm{H}), 7.19-7.23(\mathrm{~m}, 3 \mathrm{H}), 7.28-7.32(\mathrm{~m}, 2 \mathrm{H}), 7.34-7.36(\mathrm{~m}, 2 \mathrm{H}), 7.77-7.83(\mathrm{~m}$, 2H). LC - MS (ESI) (m/z) $390.1(\mathrm{M}+1)^{+}$.

5-((4-benzhydrylpiperazin-1-yl)methyl)-3-(4-fluorobenzyl)oxazolidin-2-one (4h)

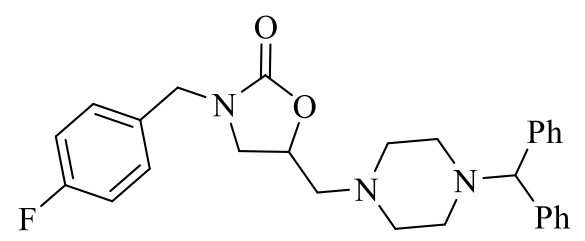

Preparation of 5-((4-benzhydrylpiperazin-1-yl)methyl)-3-(4-fluorobenzyl) oxazolidin-2-one (4h): (3-(4-fluorobenzyl)-2-oxooxazolidin-5-yl)methyl 4-methylbenzene sulfonate (1.1215 g, 2.955 
mmol) and diphenylmethylpiperazine and (1.49 g, $5.90 \mathrm{mmol})$ in anhydrous tetrahydrofuran (5 $\mathrm{mL})$ and triethyl amine $(1.1648 \mathrm{~g}, 11.51 \mathrm{mmol})$ were stirred under microwave at $1200 \mathrm{C}$ for 1 hour. After 1 hour, the solvent was stripped off, residue was dissolved in dichloromethane and purified by silica gel column using hexane- ethyl acetate (0-100\%) to obtain the desired product (0.8941 g, 65.83 \% yield). 1H NMR: $\{\mathrm{CDCl} 3,400 \mathrm{MHz}, \delta(\mathrm{ppm})\} 2.37-2.67(\mathrm{~m}, 10 \mathrm{H}), 3.17-$ $3.19(\mathrm{~m}, 1 \mathrm{H}), 3.43(\mathrm{t}, 1 \mathrm{H}), 4.21(\mathrm{~s}, 1 \mathrm{H}), 4.39(\mathrm{~s}, 2 \mathrm{H}), 4.56-4.69(\mathrm{~m}, 1 \mathrm{H}), 7.01-7.07(\mathrm{~m}, 2 \mathrm{H})$, $7.17-7.21(\mathrm{~m}, 2 \mathrm{H}), 7.25-7.30(\mathrm{~m}, 6 \mathrm{H}), 7.40-7.42(\mathrm{~m}, 4 \mathrm{H})$. LC - MS (ESI) (m/z) 460.2 $(\mathrm{M}+1)+$.

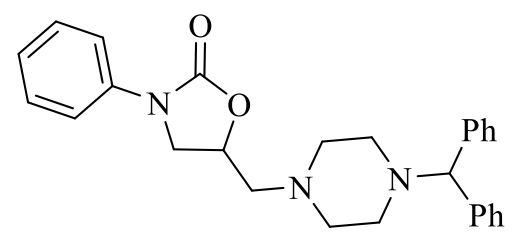

5-((4-benzhydrylpiperazin-1-yl)methyl)-3-phenyloxazolidin-2-one (4f) was prepared according to the method of 5-((4-benzhydrylpiperazin-1-yl)methyl)-3-(4-fluorobenzyl) oxazolidin-2-one (4h) except that (3-(4-fluorobenzyl)-2-oxooxazolidin-5-yl)methyl 4-methylbenzene sulfonate was replaced with (2-oxo-3-phenyloxazolidin-5-yl)methyl 4-methylbenzenesulfonate. Yield: $24.63 \% .{ }^{1} \mathrm{H}$ NMR: $\left\{\mathrm{CDCl}_{3}, 400 \mathrm{MHz}, \delta(\mathrm{ppm})\right\} 2.46-2.80(\mathrm{~m}, 10 \mathrm{H}), 3.76-3.80(\mathrm{~m}, 1 \mathrm{H}), 4.02$ (t, 1H), $4.27(\mathrm{~s}, 1 \mathrm{H}), 4.71-4.77(\mathrm{~m}, 1 \mathrm{H}), 7.14-7.17(\mathrm{~m}, 1 \mathrm{H}), 7.20-7.24(\mathrm{~m}, 2 \mathrm{H}), 7.29-7.33$ $(\mathrm{m}, 4 \mathrm{H}), 7.37-7.42(\mathrm{~m}, 2 \mathrm{H}), 7.45-7.47(\mathrm{~m}, 4 \mathrm{H}), 7.56-7.58(\mathrm{~m}, 2 \mathrm{H}) . \mathrm{LC}-\mathrm{MS}(\mathrm{ESI})(\mathrm{m} / \mathrm{z})$ $428.2(\mathrm{M}+1)^{+}$.

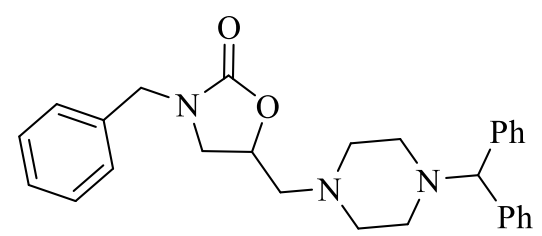

5-(4-benzhydryl-piperazin-1-ylmethyl)-3-benzyl-oxazolidin-2-one (4g) was prepared according to the method of 5-((4-benzhydrylpiperazin-1-yl)methyl)-3-(4-fluorobenzyl) oxazolidin-2-one 
(4h) except that (3-(4-fluorobenzyl)-2-oxooxazolidin-5-yl)methyl 4-methylbenzene sulfonate was replaced with (3-benzyl-2-oxooxazolidin-5-yl)methyl 4-methylbenzenesulfonate. Yield: $44.7 \% .{ }^{1} \mathrm{H}-\mathrm{NMR}\left\{\mathrm{CDCl}_{3}, 400 \mathrm{MHz}, \delta(\mathrm{ppm})\right\} 2.50(\mathrm{bs}, 4 \mathrm{H}), 2.71-2.95(\mathrm{~m}, 6 \mathrm{H}), 3.05-3.09$ (m, 1H), $3.50(\mathrm{t}, 1 \mathrm{H}), 3.99-4.13$ (heptet, 1H), $4.24(\mathrm{~s}, 1 \mathrm{H}), 4.39(\mathrm{~d}, 2 \mathrm{H}), 4.73-4.80(\mathrm{~m}, 1 \mathrm{H})$, 7.15 - $7.22(\mathrm{~m}, 2 \mathrm{H}), 7.24-7.41(\mathrm{~m}, 13 \mathrm{H}) . \mathrm{LC}-\mathrm{MS}(\mathrm{ESI})(\mathrm{m} / \mathrm{z}) 442.1(\mathrm{M}+1)^{+}$.

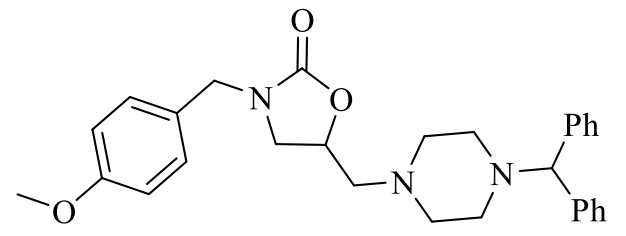

5-((4-benzhydrylpiperazin-1-yl)methyl)-3-(4-methoxybenzyl)oxazolidin-2-one (4i) was prepared according to the method of 5-((4-benzhydrylpiperazin-1-yl)methyl)-3-(4-fluorobenzyl) oxazolidin-2-one (4h) except that (3-(4-fluorobenzyl)-2-oxooxazolidin-5-yl)methyl 4methylbenzene sulfonate was replaced with (3-(4-methoxybenzyl)-2-oxooxazolidin-5-yl)methyl 4-methylbenzenesulfonate. Yield: $79.72 \% .{ }^{1} \mathrm{H}$ NMR: $\left\{\mathrm{CDCl}_{3}, 400 \mathrm{MHz}, \delta(\mathrm{ppm})\right\} 2.38-2.67$ (m, 10H), $3.12-3.15(\mathrm{~m}, 1 \mathrm{H}), 3.42(\mathrm{t}, 1 \mathrm{H}), 3.82(\mathrm{~s}, 3 \mathrm{H}), 4.21(\mathrm{~s}, 1 \mathrm{H}), 4.31-4.40(\mathrm{~s}, 2 \mathrm{H}), 4.56-$ $4.62(\mathrm{~m}, 1 \mathrm{H}), 6.87-6.91(\mathrm{~m}, 2 \mathrm{H}), 7.16-7.23(\mathrm{~m}, 4 \mathrm{H}), 7.27-7.29(\mathrm{~m}, 4 \mathrm{H}), 7.40-7.41(\mathrm{~m}$, 4H). LC - MS (ESI) (m/z) $472.2(\mathrm{M}+1)^{+}$.

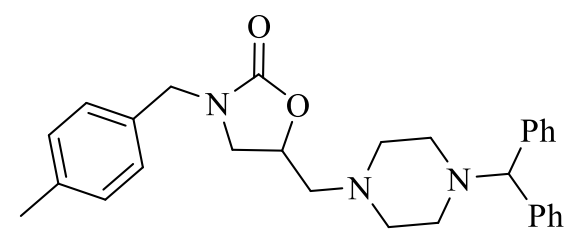

5-((4-benzhydrylpiperazin-1-yl)methyl)-3-(4-methylbenzyl)oxazolidin-2-one (4j) was prepared according to the method of 5-((4-benzhydrylpiperazin-1-yl)methyl)-3-(4-fluorobenzyl) oxazolidin-2-one (4h) except that (3-(4-fluorobenzyl)-2-oxooxazolidin-5-yl)methyl 4methylbenzene sulfonate was replaced with (3-(4-Methylbenzyl)-2-oxooxazolidin-5-yl)methyl 4methylbenzenesulfonate. Yield: $73.95 \% .{ }^{1} \mathrm{H}$ NMR: $\left\{\mathrm{CDCl}_{3}, 400 \mathrm{MHz}, \delta(\mathrm{ppm})\right\} 2.37-2.68(\mathrm{~m}$, 
$13 \mathrm{H}), 3.13-3.17(\mathrm{~m}, 1 \mathrm{H}), 3.43(\mathrm{t}, 1 \mathrm{H}), 4.21(\mathrm{~s}, 1 \mathrm{H}), 4.34-4.43(\mathrm{~m}, 2 \mathrm{H}), 4.56-4.63(\mathrm{~m}, 1 \mathrm{H})$, $7.17-7.21(\mathrm{~m}, 6 \mathrm{H}), 7.26-7.30(\mathrm{~m}, 4 \mathrm{H}), 7.40-7.42(\mathrm{~m}, 4 \mathrm{H}) . \mathrm{LC}-\mathrm{MS}(\mathrm{ESI})(\mathrm{m} / \mathrm{z}) 456.3$ $(\mathrm{M}+1)^{+} .5-((4-$

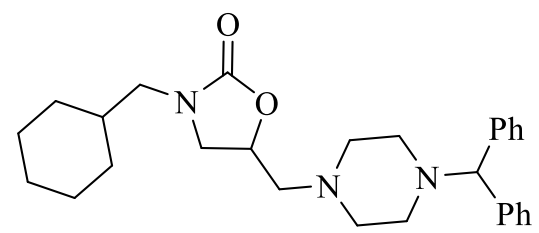

Benzhydrylpiperazin-1-yl)methyl)-3-(cyclohexylmethyl)oxazolidin-2-one (4k) was prepared according to the method of 5-((4-benzhydrylpiperazin-1-yl)methyl)-3-(4-fluorobenzyl) oxazolidin-2-one (4h) except that (3-(4-fluorobenzyl)-2-oxooxazolidin-5-yl)methyl 4methylbenzene sulfonate was replaced with (3-(cyclohexylmethyl)-2-oxooxazolidin-5-yl)methyl 4-methylbenzenesulfonate. Yield: $66.45 \% .{ }^{1} \mathrm{H} \mathrm{NMR}:\left\{\mathrm{CDCl}_{3}, 400 \mathrm{MHz}, \delta(\mathrm{ppm})\right\} 0.92-1.01$ $(\mathrm{m}, 2 \mathrm{H}), 1.12-1.29(\mathrm{~m}, 3 \mathrm{H}), 1.52-1.76(\mathrm{~m}, 7 \mathrm{H}), 2.42-2.70(\mathrm{~m}, 10 \mathrm{H}), 3.02-3.11(\mathrm{~m}, 2 \mathrm{H})$, $3.28-3.32(\mathrm{~m}, 1 \mathrm{H}), 3.57(\mathrm{t}, 1 \mathrm{H}), 4.22(\mathrm{~s}, 1 \mathrm{H}), 4.58-4.70(\mathrm{~m}, 1 \mathrm{H}), 7.17-7.21(\mathrm{~m}, 2 \mathrm{H}), 7.26-$ $7.30(\mathrm{~m}, 4 \mathrm{H}), 7.40-7.42(\mathrm{~m}, 4 \mathrm{H}) . \mathrm{LC}-\mathrm{MS}(\mathrm{ESI})(\mathrm{m} / \mathrm{z}) 448.3(\mathrm{M}+1)^{+}$.<smiles>O=C1OC(CN2CCN(C(c3ccccc3)c3ccccc3)CC2)CN1CC1CCOCC1</smiles>

5-(4-Benzhydryl-piperazin-1-ylmethyl)-3-(tetrahydro-pyran-4-ylmethyl)-oxazolidin-2-one was prepared according to the method of 5-((4-benzhydrylpiperazin-1-yl)methyl)-3-(4fluorobenzyl) oxazolidin-2-one (4h) except that (3-(4-fluorobenzyl)-2-oxooxazolidin-5yl)methyl 4-methylbenzene sulfonate was replaced with (2-oxo-3-((tetrahydro-2H-pyran-4yl)methyl)oxazolidin-5-yl)methyl 4-methylbenzene sulfonate. Yield: $75.85 \%$. ${ }^{1} \mathrm{H}$ NMR: $\left\{\mathrm{CDCl}_{3}, 400 \mathrm{MHz}, \delta(\mathrm{ppm})\right\} 1.31-1.41(\mathrm{~m}, 2 \mathrm{H}), 1.58-1.61(\mathrm{~m}, 2 \mathrm{H}), 1.74(\mathrm{~s}, 1 \mathrm{H}), 1.81-1.92$ (m, 1H), $2.41-2.70(\mathrm{~m}, 10 \mathrm{H}), 3.08-3.18(\mathrm{~m}, 2 \mathrm{H}), 3.31-3.41(\mathrm{~m}, 3 \mathrm{H}), 3.60(\mathrm{t}, 1 \mathrm{H}), 3.97-$ 
$4.01(\mathrm{~m}, 2 \mathrm{H}), 4.22(\mathrm{~s}, 1 \mathrm{H}), 4.60-4.66(\mathrm{~m}, 1 \mathrm{H}), 7.17-7.21(\mathrm{~m}, 2 \mathrm{H}), 7.26-7.30(\mathrm{~m}, 4 \mathrm{H}), 7.40-$ $7.42(\mathrm{~m}, 4 \mathrm{H}) . \mathrm{LC}-\mathrm{MS}(\mathrm{ESI})(\mathrm{m} / \mathrm{z}) 450.3(\mathrm{M}+1)^{+}$.

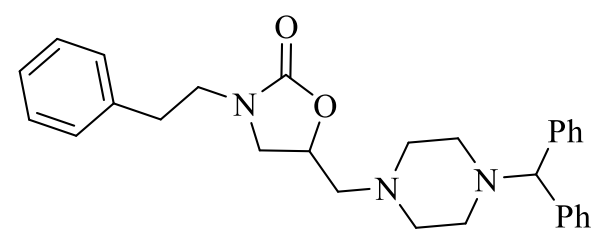

5-((4-benzhydrylpiperazin-1-yl)methyl)-3-phenethyloxazolidin-2-one (4m) was prepared according to the method of 5-((4-benzhydrylpiperazin-1-yl)methyl)-3-(4-fluorobenzyl) oxazolidin-2-one (4h) except that (3-(4-fluorobenzyl)-2-oxooxazolidin-5-yl)methyl 4methylbenzene sulfonate was replaced with (2-oxo-3-phenethyloxazolidin-5-yl)methyl 4methylbenzenesulfonate. Yield: $70.75 \% .{ }^{1} \mathrm{H}$ NMR: $\{\mathrm{CDCl} 3,400 \mathrm{MHz}, \delta(\mathrm{ppm})\} 2.40-2.63(\mathrm{~m}$, $10 \mathrm{H}), 2.87-2.91(\mathrm{~m}, 2 \mathrm{H}), 3.12-3.16(\mathrm{~m}, 1 \mathrm{H}), 3.42(\mathrm{t}, 1 \mathrm{H}), 3.51(\mathrm{t}, 1 \mathrm{H}), 4.22(\mathrm{~s}, 1 \mathrm{H}), 4.50-$ $4.57(\mathrm{~m}, 1 \mathrm{H}), 7.17-7.33(\mathrm{~m}, 11 \mathrm{H}), 7.40-7.42(\mathrm{~m}, 4 \mathrm{H}) . \mathrm{LC}-\mathrm{MS}(\mathrm{ESI})(\mathrm{m} / \mathrm{z}) 456.2(\mathrm{M}+1)^{+}$.

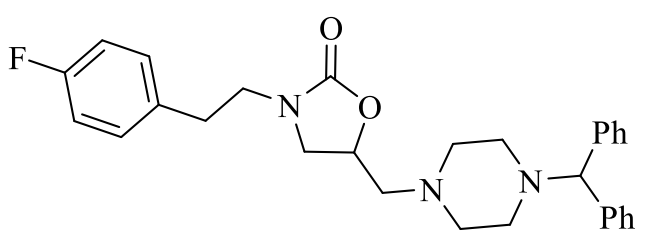

5-((4-benzhydrylpiperazin-1-yl)methyl)-3-(4-fluorophenethyl)oxazolidin-2-one (4n) was prepared according to the method of 5-((4-benzhydrylpiperazin-1-yl)methyl)-3-(4-fluorobenzyl) oxazolidin-2-one (4h) except that (3-(4-fluorobenzyl)-2-oxooxazolidin-5-yl)methyl 4methylbenzene sulfonate was replaced with (3-(4-fluorophenethyl)-2-oxooxazolidin-5-yl)methyl 4-methylbenzenesulfonate. Yield: 59.36\% . ${ }^{1} \mathrm{H}$ NMR: $\{\mathrm{CDCl} 3,400 \mathrm{MHz}, \delta(\mathrm{ppm})\} 2.30-2.53$ $(\mathrm{m}, 10 \mathrm{H}), 2.75(\mathrm{t}, 2 \mathrm{H}), 3.03-3.07(\mathrm{~m}, 1 \mathrm{H}), 3.30-3.41(\mathrm{~m}, 3 \mathrm{H}), 4.12(\mathrm{~s}, 1 \mathrm{H}), 4.40-4.47(\mathrm{~m}$, 1H), $6.87-6.92(\mathrm{~m}, 2 \mathrm{H}), 7.05-7.10(\mathrm{~m}, 4 \mathrm{H}), 7.16-7.19(\mathrm{~m}, 4 \mathrm{H}), 7.30-7.32(\mathrm{~m}, 4 \mathrm{H}) . \mathrm{LC}-$ $\operatorname{MS}(\mathrm{ESI})(\mathrm{m} / \mathrm{z}) 474.2(\mathrm{M}+1)^{+}$. 


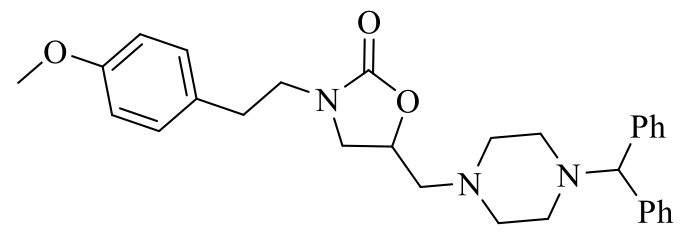

5-(4-Benzhydryl-piperazin-1-ylmethyl)-3-[2-(4-methoxy-phenyl)-ethyl]-oxazolidin-2-one

was prepared according to the method of 5-((4-benzhydrylpiperazin-1-yl)methyl)-3-(4fluorobenzyl) oxazolidin-2-one (4h) except that (3-(4-fluorobenzyl)-2-oxooxazolidin-5yl)methyl 4-methylbenzene sulfonate was replaced with (3-(4-methoxyphenethyl)-2oxooxazolidin-5-yl)methyl 4-methylbenzenesulfonate. Yield: $48.17 \%$. ${ }^{1} \mathrm{H}$ NMR: $\{\mathrm{CDCl} 3,400$ MHz, $\delta(\mathrm{ppm})\} 2.41-2.63(\mathrm{~m}, 10 \mathrm{H}), 2.82(\mathrm{t}, 2 \mathrm{H}), 3.12-3.16(\mathrm{~m}, 1 \mathrm{H}), 3.40-3.49(\mathrm{~m}, 3 \mathrm{H})$, $3.79(\mathrm{~s}, 3 \mathrm{H}), 4.23(\mathrm{~m}, 1 \mathrm{H}), 4.50-4.57(\mathrm{~m}, 1 \mathrm{H}), 6.84-6.87(\mathrm{~m}, 2 \mathrm{H}), 7.13-7.21(\mathrm{~m}, 4 \mathrm{H}), 7.27-$ $7.30(\mathrm{~m}, 4 \mathrm{H}), 7.41-7.43(\mathrm{~m}, 4 \mathrm{H}) . \mathrm{LC}-\mathrm{MS}(\mathrm{ESI})(\mathrm{m} / \mathrm{z}) 486.3(\mathrm{M}+1)^{+}$.

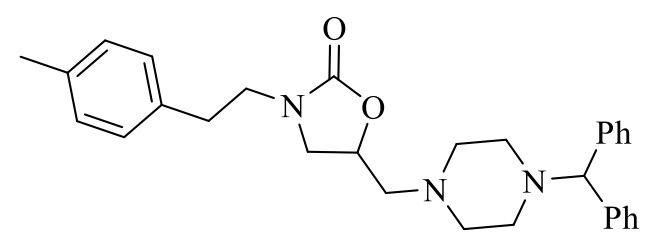

5-(4-Benzhydryl-piperazin-1-ylmethyl)-3-(2-p-tolyl-ethyl)-oxazolidin-2-one (4p) was prepared according to the method of 5-((4-benzhydrylpiperazin-1-yl)methyl)-3-(4-fluorobenzyl) oxazolidin-2-one (4h) except that (3-(4-fluorobenzyl)-2-oxooxazolidin-5-yl)methyl 4methylbenzene sulfonate was replaced with (3-(4-methylphenethyl)-2-oxooxazolidin-5yl)methyl 4-methylbenzenesulfonate. Yield: $63.17 \% .{ }^{1} \mathrm{H}$ NMR: $\{\mathrm{CDCl} 3,400 \mathrm{MHz}, \delta(\mathrm{ppm})\}$ $2.23-2.53(\mathrm{~m}, 13 \mathrm{H}), 2.72-2.77(\mathrm{~m}, 2 \mathrm{H}), 3.02-3.06(\mathrm{~m}, 1 \mathrm{H}), 3.32(\mathrm{t}, 1 \mathrm{H}), 3.40(\mathrm{t}, 2 \mathrm{H}), 4.12$ (s, 1H), $4.40-4.47(\mathrm{~m}, 1 \mathrm{H}), 7.07-7.11(\mathrm{~m}, 2 \mathrm{H}), 7.16-7.20(\mathrm{~m}, 4 \mathrm{H}), 7.30-7.33(\mathrm{~m}, 4 \mathrm{H}) . \mathrm{LC}-$ MS (ESI) (m/z) $470.3(\mathrm{M}+1)^{+}$. 


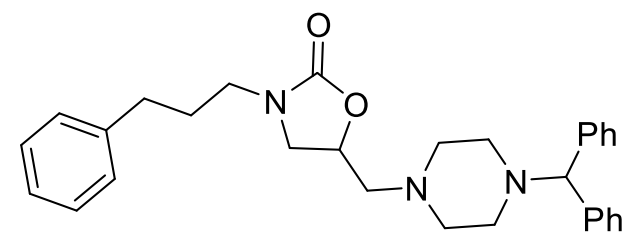

5-((4-benzhydrylpiperazin-1-yl)methyl)-3-(3-phenylpropyl)oxazolidin-2-one (4q) was prepared according to the method of 5-((4-benzhydrylpiperazin-1-yl)methyl)-3-(4-fluorobenzyl) oxazolidin-2-one (4h) except that (3-(4-fluorobenzyl)-2-oxooxazolidin-5-yl)methyl 4methylbenzene sulfonate was replaced with (2-oxo-3-(3-phenylpropyl)oxazolidin-5-yl)methyl 4methylbenzenesulfonate. Yield: $24.79 \% .{ }^{1} \mathrm{H}$ NMR: $\left\{\mathrm{CDCl}_{3}, 400 \mathrm{MHz}, \delta(\mathrm{ppm})\right\} 1.75-1.82$ (pentet, 2H), $2.31-2.58(\mathrm{~m}, 12 \mathrm{H}), 3.13-3.27(\mathrm{~m}, 3 \mathrm{H}), 3.43(\mathrm{t}, 1 \mathrm{H}), 4.10(\mathrm{~s}, 1 \mathrm{H}), 4.44-4.50$ $(\mathrm{m}, 1 \mathrm{H}), 7.07-7.14(\mathrm{~m}, 5 \mathrm{H}), 7.16-7.23(\mathrm{~m}, 6 \mathrm{H}), 7.30-7.32(\mathrm{~m}, 4 \mathrm{H}) . \mathrm{LC}-\mathrm{MS}(\mathrm{ESI})(\mathrm{m} / \mathrm{z})$ 470.3

Computational values: TPSA and cLogP values were calculated using the Dotmatics software suite (Dotmatics LLC The Old Monastery, Windhill Bishops, Stortford Herts, CW23 2ND UK). Sigma-1 and sigma-2 competitive radioligand-binding studies: Competitive binding assays were conducted by the Psychoactive Drug Screening Program (PDSP) at The University of North Carolina, Chapel Hill under the direction of Professor Bryan Roth. Assay conditions can be found in the PDSP assay protocol book at https://pdsp.unc.edu/pdspweb/content/UNCCH\%20Protocol\%20Book.pdf.<smiles>[Z19]CC1CN([R])C(=O)O1</smiles> 
Table 1. In vitro screening and physicochemical properties data for $(\mathbf{4 a})-(\mathbf{4 q})$

\begin{tabular}{|c|c|c|c|c|c|c|c|}
\hline \multirow{2}{*}{ Entry } & \multirow{2}{*}{$\mathbf{R}$} & \multirow{2}{*}{ MW } & \multirow{2}{*}{ cLogP } & \multirow{2}{*}{ TPSA } & $\sigma_{2}$ & $\sigma_{1}$ & \multirow{2}{*}{$\begin{array}{l}\sigma_{1} / \sigma_{2} \\
\text { Ratio }\end{array}$} \\
\hline & & & & & \multicolumn{2}{|c|}{$\mathbf{K}_{\mathbf{i}}(\mathbf{n M})$} & \\
\hline $4 a$ & $\mathrm{H}$ & 351 & 2.45 & 45 & 10000 & 10000 & NA \\
\hline $4 \mathrm{~b}$ & Cyclopropyl & 392 & 3.59 & 36 & 119 & 10000 & 84 \\
\hline $4 \mathrm{~d}$ & Cyclobutyl & 406 & 4.04 & 36 & 465 & 10000 & 22 \\
\hline $4 c$ & Cyclopentyl & 420 & 4.49 & 36 & 206 & 10000 & 49 \\
\hline $4 \mathrm{e}$ & Cyclohexyl & 434 & 4.94 & 36 & 530 & 10000 & 19 \\
\hline $4 \mathrm{f}$ & $\mathrm{Ph}$ & 428 & 4.63 & 36 & 192 & 10000 & 52 \\
\hline $4 \mathrm{~g}$ & $\mathrm{CH}_{2} \mathrm{Ph}$ & 442 & 4.75 & 36 & 91 & 10000 & 110 \\
\hline $4 \mathrm{~h}$ & $\mathrm{CH}_{2}(4-\mathrm{F}-\mathrm{Ph})$ & 460 & 4.85 & 36 & 36 & 2847 & 79 \\
\hline $4 \mathrm{i}$ & $\mathrm{CH}_{2}(4-\mathrm{OMe}-\mathrm{Ph})$ & 472 & 4.71 & 45 & 10000 & 10000 & $\mathrm{NA}$ \\
\hline $4 \mathrm{j}$ & CH2(4-Me-Ph) & 456 & 5.03 & 36 & 10000 & 10000 & NA \\
\hline $4 \mathrm{k}$ & $\mathrm{CH}_{2}$ Cyclohexyl & 448 & 5.16 & 36 & 1379 & 10000 & 7 \\
\hline 41 & $\mathrm{CH}_{2}$-THP & 450 & 3.7 & 45 & 2428 & 10000 & 4 \\
\hline $4 \mathrm{~m}$ & $\mathrm{CH}_{2} \mathrm{CH}_{2} \mathrm{Ph}$ & 456 & 4.85 & 36 & 49 & 10000 & 204 \\
\hline $4 n$ & $\mathrm{CH}_{2} \mathrm{CH}_{2}(4-\mathrm{F}-\mathrm{Ph})$ & 474 & 4.95 & 36 & 2338 & 10000 & 4 \\
\hline 40 & $\mathrm{CH}_{2} \mathrm{CH}_{2}(4-\mathrm{OMe}-\mathrm{Ph})$ & 486 & 4.81 & 45 & 10000 & 10000 & $\mathrm{NA}$ \\
\hline $4 p$ & $\mathrm{CH}_{2} \mathrm{CH}_{2}(4-\mathrm{Me}-\mathrm{Ph})$ & 470 & 5.13 & 36 & 10000 & 10000 & NA \\
\hline $4 q$ & $\mathrm{CH}_{2} \mathrm{CH}_{2} \mathrm{CH}_{2} \mathrm{Ph}$ & 470 & 5.3 & 36 & 1245 & 10000 & 8 \\
\hline
\end{tabular}

Table 2: Off-target assessment of (4h) at key CNS targets

\begin{tabular}{|c|c|c|c|c|c|c|c|c|c|}
\hline $\boldsymbol{\sigma}_{\mathbf{2}}$ & $\boldsymbol{\sigma}_{\mathbf{1}}$ & $\boldsymbol{\alpha}_{1 \mathrm{~A}}$ & $\boldsymbol{\alpha}_{\mathbf{1 B}}$ & $\boldsymbol{\alpha}_{\mathbf{1 D}}$ & $\boldsymbol{\alpha}_{2 \mathrm{~A}}$ & $\boldsymbol{\alpha}_{\mathbf{2 B}}$ & $\boldsymbol{\alpha}_{2 \mathrm{C}}$ & $\boldsymbol{\beta}_{\mathbf{1}}$ & $\boldsymbol{\beta}_{\mathbf{2}}$ \\
\hline 36 & 2847 & 1705 & 10000 & 10000 & 10000 & 1136 & 2159 & 10000 & 10000 \\
\hline
\end{tabular}




\begin{tabular}{|c|c|c|c|c|c|c|c|c|c|}
\hline$\beta_{3}$ & BZP-B & BZP-P & $\mathrm{D}_{1}$ & $\mathrm{D}_{2}$ & $\mathrm{D}_{3}$ & $\mathrm{D}_{4}$ & $\mathrm{D}_{5}$ & DAT & DOP \\
\hline 10000 & 10000 & 10000 & 10000 & 1508 & 1293 & 521 & 10000 & 186 & 10000 \\
\hline $\mathrm{GABA}_{\mathrm{A}}$ & $\mathrm{H}_{1}$ & $\mathrm{H}_{2}$ & $\mathrm{H}_{3}$ & $5 \mathrm{HT}_{1 \mathrm{~A}}$ & $5 \mathrm{HT}_{1 \mathrm{~B}}$ & $5 \mathrm{HT}_{1 \mathrm{D}}$ & $5 \mathrm{HT}_{1 \mathrm{E}}$ & $5 \mathrm{HT}_{2 \mathrm{~A}}$ & $5 \mathrm{HT}_{2 \mathrm{~B}}$ \\
\hline 10000 & 99 & 10000 & 10000 & 10000 & 10000 & 5565 & 10000 & 1072 & 201 \\
\hline $5 \mathrm{HT}_{2 \mathrm{C}}$ & $\mathrm{KOP}$ & $\mathrm{M}_{1}$ & $\mathrm{M}_{2}$ & $\mathrm{M}_{3}$ & $\mathrm{M}_{4}$ & $\mathrm{M}_{5}$ & $\mathrm{MOP}$ & $\mathrm{NET}$ & $\mathrm{SERT}$ \\
\hline 10000 & 1007 & 10000 & 10000 & 10000 & 10000 & 10000 & 10000 & 967 & 10000 \\
\hline
\end{tabular}

transporter, $\mathrm{DOP}=\delta$-opioid receptor, $\mathrm{KOP}=\kappa$-opioid receptor, $\mathrm{MOP}=\mu$-opioid receptor, $\mathrm{NET}=$ norepinephrine transporter, SERT $=$ serotonin transporter

Acknowledgments: $\mathrm{K}_{\mathrm{i}}$ determinations for compound binding to Sigma-1, and Sigma-2 were generously provided by the National Institute of Mental Health's Psychoactive Drug Screening Program, Contract \# HHSN-271-2013-00017-C (NIMH PDSP). The NIMH PDSP is directed by Bryan L. Roth at the University of North Carolina at Chapel Hill and Project Officer Jamie Driscoll at NIMH, Bethesda MD, USA. For experimental details please refer to the PDSP web site https://pdsp.unc.edu/ims/investigator/web/. The content of this manuscript is solely the responsibility of the authors and does not necessarily represent the official views of the National Institutes of Health. TPSA and cLogP values were generated using the Dotmatics software suite (Dotmatics LLC The Old Monastery, Windhill Bishops, Stortford Herts, CW23 2ND UK).

Declaration of interests: Drs. Blass and Canney both have equity interests in Praeventix LLC, which have been reviewed and approved by Temple University in accordance with its conflict of interest policies. Questions regarding this interest may be directed to the Temple University Conflict of Interest Program. No other author has reported conflicts of interest to disclose at the time of publication. 


\section{References and notes}

1. Hippius H, Neundörfer G. The discovery of Alzheimer's disease. Dialogues Clin Neurosci. 2003;5(1):101-108. doi:10.31887/DCNS.2003.5.1/hhippius

2. https://www.alz.org/alzheimers-dementia/facts-figures

3. Selkoe DJ, Hardy J: The amyloid hypothesis of Alzheimer's disease at 25 years. EMBO Mol Med 2016:1-14 doi:10.15252/emmm.201606210

4. Hawkes N: Merck ends trial of potential Alzheimer's drug verubecestat. BMJ 2017; 356:j845 doi: https://doi.org/10.1136/bmj.j845

5. Henley DB, May PC, Dean RA, Siemers ER. Development of semagacestat (LY450139), a functional gamma-secretase inhibitor, for the treatment of Alzheimer's disease. Expert Opin Pharmacother. 2009 Jul;10(10):1657-64. doi: 10.1517/14656560903044982. PMID: 19527190.

6. (a) Salloway S, Sperling R, Fox NC, Blennow K, Klunk W, Raskind M, Sabbagh M, Honig LS, Porsteinsson AP, Ferris S, Reichert M, Ketter N, Nejadnik B, Guenzler V, Miloslavsky M, Wang D, Lu Y, Lull J, Tudor IC, Liu E, Grundman M, Yuen E, Black R, Brashear HR: Two phase 3 trials of bapineuzumab in mild-to-moderate Alzheimer's disease. N Engl J Med 2014; 370:322-33 DOI: 10.1056/NEJMoa1304839 (b) Doody RS, Thomas RG, Farlow M, Iwatsubo T, Vellas B, Joffe S, Kieburtz K, Raman R, Sun X, Aisen PS, Siemers E, Liu-Seifert H, Mohs R: Phase 3 trials of solanezumab for mild-tomoderate Alzheimer's disease. N Engl J Med 2014; 370:311-21 DOI:

10.1056/NEJMoa1312889

7. https://www.fda.gov/drugs/postmarket-drug-safety-information-patients-andproviders/aducanumab-marketed-aduhelm-information accessed 10/06/2021 
8. (a) Viola KL, Klein WL: Amyloid beta oligomers in Alzheimer's disease pathogenesis, treatment, and diagnosis. Acta Neuropathol 2015;129:183-206. DOI: 10.1007/s00401015-1386-3 (b) Selkoe DJ, Hardy J: The amyloid hypothesis of Alzheimer's disease at 25 years. EMBO Mol Med 2016:1-14 doi:10.15252/emmm.201606210

9. Jonsson T, Atwal JK, Steinberg S, Snaedal J, Jonsson PV, Bjornsson S, Stefansson H, Sulem P, Gudbjartsson D, Maloney J, Hoyte K, Gustafson A, Liu Y, Lu Y, Bhangale T, Graham RR, Huttenlocher J, Bjornsdottir G, Andreassen OA, Jönsson EG, Palotie A, Behrens TW, Magnusson OT, Kong A, Thorsteinsdottir U, Watts RJ, Stefansson K A mutation in APP protects against Alzheimer's disease and age-related cognitive decline. Nature. 2012;488:96-99. https://doi.org/10.1038/nature11283

10. Limegrover CS, LeVine H, Izzo NJ, et al. Alzheimer's protection effect of A673T mutation may be driven by lower $\mathrm{A} \beta$ oligomer binding affinity. J Neurochem. 2020 . https://doi.org/10.1111/jnc.15212. jnc.15212.

11. (a) Viola KL, Klein WL: Amyloid beta oligomers in Alzheimer's disease pathogenesis, treatment, and diagnosis. Acta Neuropathol 2015;129:183-206 (b) Xia M, Cheng X, Yi R, Gao D, Xiong J: The Binding Receptors of A $\beta$ : an Alternative Therapeutic Target for Alzheimer's Disease. Mol Neurobiol 2014 doi:10.1007/s12035-014-8994-0 (c) Goure WF, Krafft G a, Jerecic J, Hefti F: Targeting the proper amyloid-beta neuronal toxins: a path forward for Alzheimer's disease immunotherapeutics. Alzheimers Res Ther 2014; 6:42 doi: 10.1186/alzrt272.

12. (a) Su TP, Evidence for sigma opioid receptor: binding of [3H]SKF-10047 to etorphine inaccessible sites in guinea-pig brain. J. Pharmacol. Exp. Ther. 1982;223(2):284-90. doi.org/10.1007/s00044-020-02574-9 (b) Khazan N, Young GA, El-Fakany EE, Hong O, 
Caliigaro D, Sigma receptors mediated the psychotomimetic effects of Nallylnormetazocine (SKF-10,047), but not its opioid agonistic-antagonistic properties. Neuropharma. 1984;23(8):983-987. DOI: 10.1016/0028-3908(84)90015-7

13. Bartz F, Kern L, Erz D, Zhu M, Gilbert D, Meinhof T, Wirkner U, Erfle H, Muckenthaler M, Pepperkok R, Runz H, Identification of cholesterol-regulating genes by targeted RNAi screening. Cell Metab. 2009;10(1):63-75. doi: 10.1016/j.cmet.2009.05.009

14. Ebrahimi-Fakhar D, Wahlster L, Bartz F, Werenbeck-Ueding J, Praggastis M, Zhang J, Joggerst-Thomalla B, Theiss S, Grimm D, Ory DS, Runz H. Reduction of TMEM97 increases NPC1 protein levels and restores cholesterol trafficking in Niemann-pick type C1 disease cells. Hum Mol Genet. 2016;15;25(16):3588-3599. doi:10.1093/hmg/ddw204.

15. Alon A, Schmidt HR, Wood MD, Sahn JJ, Martin SF, Krusea AC, Identification of the gene that codes for the $\sigma 2$ receptor, Proc. Natl. Acad. Sci. U. S. A. 2017;114(27):71607165. doi: 10.1073/pnas.1705154114.

16. (a) Izzo NJ, Staniszewski A, To L, Fa M, Teich AF, Saeed F, Wostein H, Walko T, Vaswani A, Wardius M, Syed Z, Ravenscroft J, Mozzoni K, Silky C, Rehak C, Yurko R, Finn P, Look G, Rishton G, Safferstein H, Miller M, Johanson C, Stopa E, Windisch M, Hutter-Paier B, Shamloo M, Arancio O, LeVine H, Catalano SM: Alzheimer's therapeutics targeting amyloid beta 1-42 oligomers I: Abeta 42 oligomer binding to specific neuronal receptors is displaced by drug candidates that improve cognitive deficits. PLoS One Edited by Wu Z-Y. 2014; 9:e111898 (b) Izzo NJ, Xu J, Zeng C, Kirk MJ, Mozzoni K, Silky C, Rehak C, Yurko R, Look G, Rishton G, Safferstein H, Cruchaga C, Goate A, Cahill MA, Arancio O, Mach RH, Craven R, Head E, LeVine H, Spires-Jones TL, Catalano SM: Alzheimer's therapeutics targeting amyloid beta 1-42 
oligomers II: Sigma-2/PGRMC1 receptors mediate Abeta 42 oligomer binding and synaptotoxicity. PLoS One Edited by Wu Z-Y. 2014; 9:e111899

17. (a) Rishton GM, Look GC, Ni ZJ, Zhang J, Wang Y, Huang Y, Wu X, Izzo NJ, LaBarbera KM, Limegrover CS, Rehak C, Yurko R, Catalano SM, Discovery of Investigational Drug CT1812, an Antagonist of the Sigma-2 Receptor Complex for Alzheimer's Disease ACS Med. Chem. Lett. 2021;12(9):1389-1395. DOI: 10.1021/acsmedchemlett.1c00048 (b) Izzo NJ, Staniszewski A, To L, Fa M, Teich AF, Saeed F, Wostein H, Walko T, Vaswani A, Wardius M, Syed Z, Ravenscroft J, Mozzoni K, Silky C, Rehak C, Yurko R, Finn P, Look G, Rishton G, Safferstein H, Miller M, Johanson C, Stopa E, Windisch M, Hutter-Paier B, Shamloo M, Arancio O, LeVine H, Catalano SM: Alzheimer's therapeutics targeting amyloid beta 1-42 oligomers I: Abeta 42 oligomer binding to specific neuronal receptors is displaced by drug candidates that improve cognitive deficits. PLoS One Edited by Wu Z-Y. 2014; 9:e111898 (b) Izzo NJ, Xu J, Zeng C, Kirk MJ, Mozzoni K, Silky C, Rehak C, Yurko R, Look G, Rishton G, Safferstein H, Cruchaga C, Goate A, Cahill MA, Arancio O, Mach RH, Craven R, Head E, LeVine H, Spires-Jones TL, Catalano SM: Alzheimer's therapeutics targeting amyloid beta 1-42 oligomers II: Sigma-2/PGRMC1 receptors mediate Abeta 42 oligomer binding and synaptotoxicity. PLoS One Edited by Wu Z-Y. 2014; 9:e111899

18. Vazquez-Rosa E, Watson MR, Sahn JJ, Hodges TR, Schroeder RE, Cintron-Perez CJ, Shin MK, Yin TC, Emery JL, Martin SF, Liebl DJ, Pieper AA, Neuroprotective Efficacy of a Sigma 2 Receptor/TMEM97 Modulator (DKR-1677) after Traumatic Brain Injury, ACS Chem Neurosci. 2019;10(3):1595-1602. doi: 10.1021/acschemneuro.8b00543. 
19. Sahn JJ, Mejia GL, Ray PR, Martin SF, Price TJ, Sigma 2 receptor/Tmem97 agonists produce long lasting antineuropathic pain effects in mice. ACS Chem Neurosci. 2017;8(8):1801-1811. doi: 10.1021/acschemneuro.7b00200.

20. Guo L, Zhen X, Sigma-2 receptor ligands: neurobiological effects. Curr. Med. Chem. 2015;22(8):989-1003. doi: 10.2174/0929867322666150114163607.

21. (a) Vilner BJ, John CS, Bowen WD, Sigma-1 and Sigma-2 receptors are expressed in a wide variety of human and rodent tumor cell lines, Cancer Res. 1995 Jan 15;55(2):40813. PMID: 7812973. (b) Asong G, Zhu XY, Bricker B, et al. New analogs of SYA013 as sigma-2 ligands with anticancer activity. Bioorg Med Chem. 2019;27(12):2629-2636. doi:10.1016/j.bmc.2019.04.012

22. Bhandare RR, Canney DJ, Bioisosteric Replacement and Related Analogs in the Design, Synthesis and Evaluation of Ligands for Muscarinic Acetylcholine Receptors, Medicinal Chemistry 2014:10(4)361-375 https://doi.org/10.2174/15734064113096660043

23. Gregory WA, Brittelli DR, Wang CLJ, Wuonola MA, McRipley RJ, Eustice DC, Eberly VS, Slee AM, Forbes M, Bartholomew PT, Antibacterials. Synthesis and structureactivity studies of 3-aryl-2-oxooxazolidines. 1. The B group, J, Med, Chem. 1989;32(8)1673-1681 https://doi-org.libproxy.temple.edu/10.1021/jm00128a003 